\section{Observational study of subclinical diabetic macular edema}

Eye (2012) 26, 900-901; doi:10.1038/eye.2012.83

Correction to: Eye (2012) 26, 833-840; doi:10.1038/eye.2012.53; published online 23 March 2012

Since the publication of this article, the authors have noticed several errors that appeared in the online version of their paper. These errors have now been rectified, and the correct article appears in this issue. The html and online pdf versions have also been rectified, and now carry the correct paper.

The corrections are listed below:

The author list was incorrect. The Diabetic Retinopathy Clinical Research Network was the author and NM Bressler, KM Miller, RW Beck, SB Bressler, AR Glassman, JW Kitchens, M Melia, and DK Schlossman comprised the writing committed. The correct author list and affiliations are shown above.

The members of the Diabetic Retinopathy Clinical Research Network who participated in the protocol were omitted. The full list is given below. The typesetters would like to apologise for these errors.

Wilmer Eye Institute, John Hopkins University School of Medicine, Baltimore, MD, USA

2Jaeb Center for Health Research, Tampa, FL, USA

${ }^{3}$ Retina and Vitreous Associates of Kentucky, Lexington, KY, USA

${ }^{4}$ Beetham Eye Institute, Joslin Diabetes Center, Boston, MA, USA

${ }^{5}$ Writing committee.

*The members of the DRCR Network who participated in the protocol are listed in the Appendix. Harvard Medical School,
Diabetic Retinopathy Clinical Research Network*, NM Bressler 1,5, KM Miller ${ }^{2,5}$, RW Beck ${ }^{2,5}$, SB Bressler 1,5, AR Glassman'2,5, JW Kitchens ${ }^{3,5}$, M Melia ${ }^{2,5}$ and DK Schlossman ${ }^{4,5}$

Coffey (V), Giorya Andreani (P), Peter Sotirakos $(\mathrm{P})$, and Terri Cain $(\mathrm{P})$.

Boston, MA, Joslin Diabetes Center (7): George S Sharuk (I), Paul G Arrigg (I), Deborah K Schlossman (I), Timothy J Murtha (I), Jennifer K Sun (I), Sabera T Shah (I), Margaret E Stockman (C, P, V), Ann Kopple (C), and Robert W Cavicchi (P).

Lexington, KY, Retina Associates of Kentucky (6): Thomas W Stone (I), John W Kitchens (I), William J Wood (I), Michelle Buck (V), Jeanne Van Arsdall (V), Judith L Cruz (V), Edward A Slade (P), and Stephen T Blevins (P).

Charlotte, NC, Charlotte Eye, Ear, Nose and Throat Assoc., P.A. (4): David Browning (I), Andrew N Antoszyk (I), Danielle R Brooks (C, V), Jennifer V Helms (C, V), Angela K Price (C, V), Melissa K Cowen (C, V), Angella S Karow (V), Heather L Murphy (V), Michael D McOwen (P), Linda M Davis (P), Loraine $M$ Clark (P), Uma M Balasubramaniam (P), Donna McClain (P), and Michele E Powers (P).

Denver, CO, Denver Health Medical Center (4): Jon M Braverman (I), Antonio P Ciardella (I), Leif S Ryman (C), Sasha I Montalvo (V), Janelle Dane Zapata (V), Rosemary C Rhodes (V), and Debbie M Brown (P).

Chicago, IL, Northwestern University Feinberg School of Medicine (3): Alice T Lyon (I), Manjot K Gill (I), Lori A Kaminski (C, V), Lori E Ackatz (C), Laima M O'Donnell (V), Jonathan Shankle (P), and Dawn M. Ryan (P). Lakeland, FL, Florida Retina Consultants (3): Scott M Friedman (I), Kelly A Blackmer (C), Jolleen S Key (C, P), Karen Sjoblom (V), Damanda A Fagan (V), Steve Carlton (P), and Allen McKinney (P).

Attleboro, MA, Southern New England Retina Associates (2): Magdalena G Krzystolik (I), Mary B Savell (C), Sandra Henriques (C, V), and Joanne E Bache (P).

Columbia, SC, Carolina Retina Center (2): Jeffrey G Gross (I), Amy M Flowers (C, V), Heidi K Lovit (V), and Randall L Price (P).
JoAnn Starr (C, V), Theresa M Butcher (C), Pamela V Singletary (V), Nancy Gore (V), Teresa 
Seattle, WA, University of Washington Medical Center (2): James L Kinyoun (I),

Susan A Rath (C, V), Brad C Clifton (P), and James D Leslie (P).

Slingerlands, NY, Retina Consultants, PLLC (2): Paul M Beer (I), Denise Garza (C), Eugenia Olmeda (C, V), Charisse Whitney (V), and Joe Fischer $(\mathrm{P})$.

Baltimore, MD, Wilmer Eye Institute at Johns Hopkins (1): Sharon D Solomon (I), Susan Bressler (I), Mary Frey $(C, V)$, Sandra West (C, V), Deborah Donohue $(\mathrm{V})$, Janis Graul (P), and David Emmert (P).

Detroit, MI, Henry Ford Health System, Department of Ophthalmology and Eye Care Services (1): Paul Andrew Edwards (I), Sheila M Rock (C, V), Janet Murphy (C, V), Brian A Rusinek (P), and Tracy A Troszak (P).

Galveston, TX, University of Texas Medical Branch, Department of Ophthalmology and Visual Sciences (1): Garvin H Davis (I), Helen K Li (I), Happy Spillar (C), John M Bourg (V), Amber Crocker (P), and Craig N Kelso (P).

Kingsport, TN, Southeastern Retina Associates, P.C. (1): Howard L Cummings (I), Deanna Jo Long (C, P), Stacy Carpenter (V), and Julie P Berry (P).

Knoxville, TN, Southeastern Retina Associates, P.C. (1): Joseph M Googe (I), Christina T Higdon (C), Cecile Hunt (V), and Paul A Blais (P).

Milwaukee, WI, Medical College of Wisconsin (1): Judy E Kim (I), Dennis P Han (I),William J Wirostko (I), Dawn Alvarez (C, V), Jeanette Graf (C), Judy Flanders $(\mathrm{V})$, Joseph R Beringer (P), and Dennis B Backes (P).

Palm Desert, CA, Southern California Desert Retina Consultants, M.C. (1): Clement K Chan (I), Kimberly S Walther (C), Sandra U Castillo (V), Kenneth M Huff (P), and Donna J Chesbrough (P).

Providence, RI, Retina Consultants (1): Caldwell W Smith (I), Robert H Janigian (I), Harold A Woodcome (I), Collin L DuCoty (C), Sylvia Varadian (C), Erika Banalewicz (V), Mark Hamel (P), and Alex L Nagle (P).

DRCR.net Coordinating Center: Jaeb Center for Health Research, Tampa, FL (staff as of 6/8/2011): Adam R Glassman (Director and Principal Investigator), Roy W Beck (Executive Director) Talat Almukhtar, Bambi J Arnold, Brian B Dale, Alyssa Baptista, Sharon R Constantine, Simone S Dupre, Allison R Edwards, Meagan L Huggins, Paula A Johnson, Lee Anne Lester, Brenda L Loggins, Emily B Malka, Shannon L McClellan, Michele Melia, Kellee M Miller, Pamela S Moke, Haijing Qin, Rosa Pritchard, Eureca Scott, and Cynthia R Stockdale.

Fundus Photograph Reading Center: University of Wisconsin-Madison, Madison, WI (staff as of $6 / 8 / 11$ ):
Matthew D Davis (Director Emeritus), Sapna Gangaputra (Co-Director), Ronald P Danis (Director and Principal Investigator), Larry Hubbard (Associate Director), James Reimers (Lead Color Photography Evaluator), Pamela Vargo (Lead Photographer), Ericka Moeller (Digital Imaging Specialist), Dawn Myers (Lead OCT Evaluator), Kristjan Burmeister (Project Manager), and Vonnie Gamma (Data Management).

DRCR.net Operations Center: Johns Hopkins University School of Medicine, Baltimore, MD (staff as of 6/8/2011): Neil M Bressler (Network Chair and Principal Investigator), Connie Lawson, Peggy R Orr, and Beth Wellman.

DRCR.net Vice Chairs: Susan B Bressler (2009-current), Scott Friedman (2009-current), Carl W Baker (2011current), and Ingrid U Scott (2009-2010).

National Eye Institute: Eleanor Schron (2009-current), Donald F Everett (2003-2006, 2007-2009), and Päivi H Miskala (2006-2007).

Executive Committee: Raj K Maturi (2009-present; Chair 2010) Neil M Bressler (2006-present; Chair 2006-2008), Lloyd Paul Aiello (2002-present; Chair 2002-2005), Carl W Baker (2009-present), Roy W Beck (2002-present), Susan B Bressler (2009-present), Alexander J Brucker (2009-present), Kakarla V Chalam (2009-present) Ronald P Danis (2004-present), Matthew D Davis (2002-present), Michael J Elman (2006-present; Chair 2009), Frederick L Ferris III (2002-present), Scott Friedman (2007-present), Adam R Glassman (2005present), Joseph Googe, Jr (2009-present), Eleanor Schron (2009-present), JoAnn Starr (2009-present), and Jennifer K Sun (2009-present). Prior Members: Andrew N Antoszyk (2009), Abdhish Bhavsar (2007-2008), David M Brown (2006-2007), David J Browning (2005-2006), Donald F Everett (2002-2009), Joan Fish (2008-2009), Andreas Lauer (2007-2008), Kim McLeod (2002-2006), Päivi H Miskala (2005-2007), Cynthia J Grinnell (2006-2007), and Ingrid U Scott (2009-2010).

Prior DRCR.net General Steering Committee Members: Neil M Bressler (Protocol Chair 2005-2008) David Browning (2005-2008), Alexander J Brucker (2005-2008), Steve Carlton (2006-2007), Emily Y Chew (2005-2008), Ronald P Danis (2003-2008), Julia A Haller (2005-2008), Lloyd Paul Aiello (2003-2008), Carl W Baker (2007-2008), Debra Paige Bunch (2007-2008), Donald F Everett (2006-2008), Frederick L Ferris III (2005-2008), Don S Fong (2003-2007), Adam R Glassman (2005-2008), Jeffrey G Gross (2006-2007), Helen K Li (2006-2007), Dennis M Marcus (2007-2008), Päivi Miskala (2005-2007), and Angela K Price (2005). 